JOURNAL CLUB

\title{
Narcolepsy risk after A/H1N1 2009 influenza vaccination
}

Vaccines are a critical tool used to prevent the of spread infectious diseases, particularly in the setting of a pandemic. While they are generally safe, rare but potentially serious adverse effects following vaccination have been reported, after licensure, for several vaccines.

This retrospective study used case coverage analysis to investigate the risk of developing narcolepsy in children receiving the AS03 adjuvanted pandemic A/H1N1 2009 influenza vaccine (Pandemrix) in England. In this study 75 children between 4-19 years of age were diagnosed with narcolepsy during the follow-up period; 11 had received the vaccine. The OR was 14.4 (95\% CI 4.3 to 48.5 ) for vaccination after adjusting for the fact that children receiving the vaccine were more likely to be in high-risk groups. The relative incidence estimate for onset of narcolepsy after vaccination using self-controlled case series analysis was 9.9 (95\% CI 2.1 to 47.9). The estimated attributable risk was between 1 in 57500 and 1 in 52000 vaccine doses.

The authors, however, point out that potential cases of narcolepsy may have been referred more quickly during the follow-up period due to a previous Finnish study reporting on the association between narcolepsy and the vaccine, thus potentially overestimating the risk in this study. Longer follow-up of the cohort exposed to the vaccine would be required to attempt to reduce this potential bias. This study shows the need for ongoing postlicensure surveillance of vaccines and also poses the question if the association is real, how might the vaccine cause narcolepsy?

\section{Simon B Drysdale}

Correspondence to Dr Simon B Drysdale, Department of Paediatrics, ST5, Epsom and St Helier University Hospitals NHS Trust, Dorking Road, Epsom, Surrey KT18 7EG, UK; simondrysdale78@yahoo.co.uk

Competing interests None.

Provenance and peer review Not commissioned; internally peer reviewed.

To cite Drysdale SB. Thorax 2014;69:389.

Published Online First 29 July 2013

Thorax 2014;69:389. doi:10.1136/thoraxjnl-2013-204079

\section{REFERENCE}

1 Miller E, Andrews N, Stellitano L, et al. Risk of narcolepsy in children and young people receiving ASO3 adjuvanted pandemic A/H1N1 2009 influenza vaccine: retrospective analysis. BMJ 2013;346:f794. 